\title{
Obstructive Sleep Apnea Severity Multiclass Classification Using Analysis of Snoring Sounds
}

\author{
Thanawan Praydas, Booncharoen Wongkittisuksa, Sawit Tanthanuch \\ Department of Electrical Engineering, Faculty of Engineering, Prince of Songkla University, \\ Hat Yai, Songkhla 90112, Thailand \\ fonthanawan.praydas@gmail.com; booncharoen.w@psu.ac.th; sawit.t@psu.ac.th
}

\begin{abstract}
The current gold standard for diagnosing obstructive sleep apnea (OSA) is an overnight multi-channel polysomnography (PSG), an expensive, labour-intensive, and uncomfortable procedure. Accordingly, it would be beneficial to have a screening method to promptly determine the severity of a patient, prior to a referral for PSG. This paper intends to distinguish the severity of OSA patients. We show that an accurate multiclass classification of snoring subjects with four classes of OSA, can be achieved on the sound spectrum of snoring without any information requirement on the number of apneas. 33 Snoring sounds with different degrees of obstructive sleep apnea and non-OSA were analyzed. The proposed technique uses K-Means clustering to cluster the sound spectrum and reconstruct features. Support vector machine (SVM) has been used for the classification. The multiclass snore sounds classification approves early stratification of subjects according to their severity. A classification accuracy of $75.76 \%$ was reported using the proposed method. The experimental results also demonstrate that the proposed method can provide diagnostic suggestions for OSA screening.
\end{abstract}

Keywords: Snoring sound, Obstructive sleep apnea, Support Vector Machine, Multiclass classification

\section{Introduction}

Obstructive Sleep Apnea (OSA) is a common sleep-related breathing disorder with significant disabling symptoms. OSA involves partial or complete collapse of the upper airway during sleep, often causing noisy breathing and heavy snoring [1]. Snoring is a common symptom of OSA, affecting more than $80 \%$ of the OSA patients [2]. OSA patients are at a higher risk of occupational and motor vehicle accidents, cardiovascular disease, and stroke [3]. Many medical researchers reported that up to $82 \%$ of men and $93 \%$ of women with moderate to severe OSA remain undiagnosed [4]. The current gold standard for diagnosing OSA is an overnight multi-channel polysomnography (PSG). However the expensive medical cost, labourintensive in setting up, and the discomfort of the electrodes attached to the head and body of the patients, are possible limitations of PSG. It would be desirable to have a non-invasive screening method with greater comfort and at a lower cost that helped respiratory physicians to promptly determine the severity of patient, prior to a referral for PSG.

In recent years, some authors have reviewed the possibility of identifying OSA through the analysis of nocturnal oximetry [5]. Acoustic analysis of snoring reveals information relating to the site and degree of obstruction of the upper airway [6]. Several studies have found the relationship between snoring and OSA using method such as snoring sounds intensity [7, 8], estimation power spectrum [9, 10], pitch detection [11, 12] and formant frequency [13]. Several of these studies have classified snoring individuals into two classes by means of AHI threshold. However, no further information about the severity of the subject is provided. The American Academy of Sleep Medicine proposes grading the severity of OSA according to the thresholds AHI $=5,15$ and $30 \mathrm{~h}^{-1}$ [14]. In this study, we assigned to classify the snoring subjects into four classes. The classes were G1 (non-OSA, AHI $<5$ ), G2 (mild OSA, 5<AHI $\leq 15$ ), G3 (moderate OSA, 15<AHI $\leq 30$ ), and G4 (severe OSA, AHI>30). This paper aims to implement K-means clustering and support vector machine (SVM) classifier for differentiate the subjects into four classes, without any requiring information on the duration or the number of apneas.

$\mathrm{K}$-means clustering is one of the most popular and widely used methods for unsupervised partitioning of data into K cluster [15, 16], we choose k-mean algorithm for clustering of the sounds spectrum, and constructed features for fed to classifier. In the last decade, support vector machines (SVM) [17] have been introduced for pattern classification and regression. SVMs based on the structural risk minimization different from the empirical risk minimization in statistical theory, we choose the SVM method to design our classification system. 
The rest parts of this paper are organized as follows. Section 2 provides the proposed approach. Section 3 presents the experimental results. Then we conclude the proposed system in Section 4.

\section{Proposed Algorithm}

In this study, the proposed system has five processing stages namely data recording, clustering, features reconstruction, classification and performance evaluation. In the data recording stage, the respiratory sounds were recorded from the Sleep Disorders Service and Research Center of Songklanagarind Hospital, Prince of Songkla University, Thailand. The sounds spectrum features were extracted from the respiratory sound signals and distinguish with K-means clustering in clustering stage. In features reconstruction stage, the abstract features were reconstructed from the sounds spectrum features and fed to the SVM classifier in the classification stage. The SVM classifier was used to differentiate non-OSA, mild OSA, moderate OSA, and severe OSA. The performance of the SVM classifier was evaluated using confusion matrix in the performance evaluation stage. The flow chart of proposed algorithm is presented in Fig. 1.

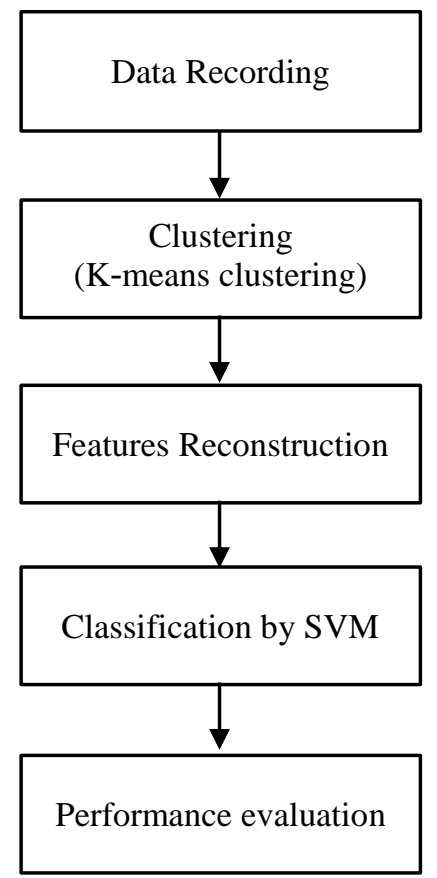

Fig. 1: Flow chart of the proposed OSA severity algorithm.

\subsection{Data Recording}

The respiratory sounds were recorded from 33 subjects (18 females, $44.7 \pm 11.6$ years), who were enrolled from the Sleep Disorders Service and Research Center Songklanagarind Hospital, Prince of Songkla University, Thailand. All were free of any diseases throughout this study. The study was approved by the hospital's research ethics commission and informed consent was obtained from all subjects. The PSG study was scored by the PSG technicians and approved by the referring physician. A total of 27 subjects, 6 were diagnosed with mild OSA, 5 with moderate OSA, 11 with severe OSA, and 5 with non-OSA. Age, gender, BMI and AHI variations of the 33 subjects are reported in Table 1. The respiratory sounds of the subjects were recorded simultaneously with PSG using a non-contact microphone. The microphone was placed $20 \mathrm{~cm}$ over the subject's head during sleep. The recordings were digitized at 10,240 Hz sampling rate and 24 bit resolution.

\subsection{Clustering}

Fig. 1 shows the flowchart of proposed OSA severity algorithm. All recorded respiratory sounds were first passed through a low pass filter with a cut-off frequency of $1,500 \mathrm{~Hz}$ to remove the effects of high frequency noise. Fast Fourier Transform (FFT) was used to calculate the sound spectrums of the respiratory sound signals. The sound spectrums of all subjects were aggregated. Then we keep $90 \%$ of the most dominant sound spectrum features from each data set. K-means clustering was 
used to divide data sets in such a way that sound spectrums belong to the same cluster. K-means clustering iteratively calculated the shortest Euclidean distance by adapting the centroid point location as shown in Eqs. (1).

Table 1: Characteristics of the study subjects.

\begin{tabular}{|l|l|l|l|l|}
\hline Class & Number of snorers & Age $(\mathrm{yr})$ & BMI $\left(\mathrm{kg} / \mathrm{m}^{2}\right)$ & AHI $\left(\mathrm{h}^{-1}\right)$ \\
\hline Non-OSA & 5 (2 females) & $52.2 \pm 11.9$ & $27.2 \pm 4.1$ & $2.8 \pm 1.1$ \\
\hline Mild OSA & 10 (8 females) & $41.3 \pm 13.5$ & $29.2 \pm 7.7$ & $9.9 \pm 3.4$ \\
\hline Moderate OSA & 7 (4 females) & $48.9 \pm 8.9$ & $24.8 \pm 3.9$ & $24.6 \pm 3.9$ \\
\hline Severe & 11 (4 females) & $41.7 \pm 10.1$ & $34.2 \pm 8.1$ & $67.2 \pm 29.8$ \\
\hline
\end{tabular}

$$
J=\sum_{j=1}^{k} \sum_{i=1}^{n}\left\|x_{i}^{(j)}-C_{j}\right\|^{2}
$$

Where $\mathrm{J}$ is the distance between the sound spectrums ( $\mathrm{x}$ ) and the cluster center (c), $\mathrm{k}$ is the number of cluster, and $\mathrm{n}$ is the number of data. The number of clusters in $\mathrm{K}$-means algorithm was set to $\mathrm{K}=500$. Our algorithm keeps the centroid cluster points in the training process.

\subsection{Feature Reconstruction}

The K-means clustering divides the sound spectrums into 500 clusters in training process. Each cluster has the sound spectrum data into $\mathrm{x}$ and $\mathrm{y}$ coordinate format. In testing process, untested sound spectrums can be mapped in the same comparing standard for reconstructed features. We compare between each cluster center and the untested sound spectrum data to find the nearest distance, as shown in Eqs. (2).

$$
d_{i j}=\sqrt{\left(x_{i}-x_{j}\right)^{2}+\left(y_{i}-y_{j}\right)^{2}}
$$

Where $d$ is the distance between the untested sound spectrum data and the trained centroid clusters. Number of sound spectrum data, which are under a cluster are combined together to get the sound spectrum density in each cluster. After this process, each cluster is represented by a single value. Each cluster value is arranged row-wise and reconstruct features which is called an abstract feature vector. In this method, the total 500 clusters are obtained from the sound signal. So the abstract feature vector has 500 values. Then these abstract features are used for training SVM classifier to obtain non-OSA, mild OSA, moderate OSA, and severe OSA.

\subsection{Support Vector Machine (SVM)}

For Classification many classifiers are used by various authors. One of the promising approach to the supervised classification scheme is a Support Vector Machine (SVM). SVM classifier is a kernel based supervised learning algorithm that classifies the data into two or more classes. During the training phase, SVM builds a model, maps the decision boundary for each class and specifies the hyperplane which separates the different classes. Increasing the distance between the classes by increasing the hyperplane margin helps to increase the classification accuracy. SVM can be used to effectively perform nonlinear classification $[15,16]$. For the classification of OSA severity, the abstract features were fed to the SVM classifier to differentiate non OSA, mild OSA, moderate OSA, and severe OSA.

\subsection{Performance Evaluation}

The confusion matrix is a performance evaluation technique used to evaluate the performance of an algorithm. As the name suggest, the confusion matrix shows the number of true and false classification which helps in evaluating the performance of the classifier [18]. The SVM classifier's performance was evaluated using confusion matrix in this study. 


\section{Results and Discussion}

The SVM classifier was performed with leave-one-out cross validation. Table 2 shows the confusion matrix of the leave-one-out cross validation of the SVM classification. The confusion matrix is a specific table layout that allows visualization of the performance of a supervised learning algorithm. Each column of the matrix represents the instances in a classification class, while each row represents the instances in an actual class. The results obtained by using the confusion matrix shows that non-OSA was miss classified as mild OSA at two instances. Mild OSA was miss classified as non-OSA at two instances and once as mderate OSA. Moderate OSA was miss classified as mild OSA at two instances and once as severe OSA. AS can be calculated from Table 2, the individual classification accuracy for non-OSA, mild OSA, moderate OSA, and severe OSA are $60 \%, 70 \%, 57.14 \%$, and $100 \%$ respectively. The accuracy classification is $75.76 \%$. Fig 2 shows the number of actual non OSA, mild OSA, moderate OSA, and severe OSA. From the confusion matrix observation, the classification of severe OSA results better accuracy and the classification of non OSA shows less accuracy compared to each class.

In this study we used the AHI values as the gold standard for grading the severity of OSA into four levels, according to the thresholds AHI $=5,15$ and $30 \mathrm{~h}^{-1}$. The limitation of AHI as the gold standard would affect the AHI cut off value. This, in turn, would affect the validation of any classification particularly for those people with marginal AHI. For example one of the misclassified instance had AHI of 4.6, this subject was grouped as non-OSA, but obviously the sound spectrum is similar to mild OSA that had AHI of 5, this subject would have been grouped as mild OSA.

Table 2: Confusion matrix of the leave-one-out cross validation of the SVM classification.

\begin{tabular}{|c|l|l|l|l|l|l|}
\hline \multirow{2}{*}{} & \multicolumn{7}{|c|}{ Classification class } \\
\cline { 2 - 7 } & & Non-OSA & Mild OSA & Moderate OSA & Severe OSA & Accuracy \\
\cline { 2 - 7 } & non-OSA & 3 & 2 & 0 & 0 & $60 \%$ \\
\cline { 2 - 7 } & mild OSA & 2 & 7 & 1 & 0 & $70 \%$ \\
\cline { 2 - 7 } & moderate OSA & 0 & 2 & 4 & 1 & $57.14 \%$ \\
\cline { 2 - 7 } & severe OSA & 0 & 0 & 0 & 11 & $100 \%$ \\
\hline
\end{tabular}

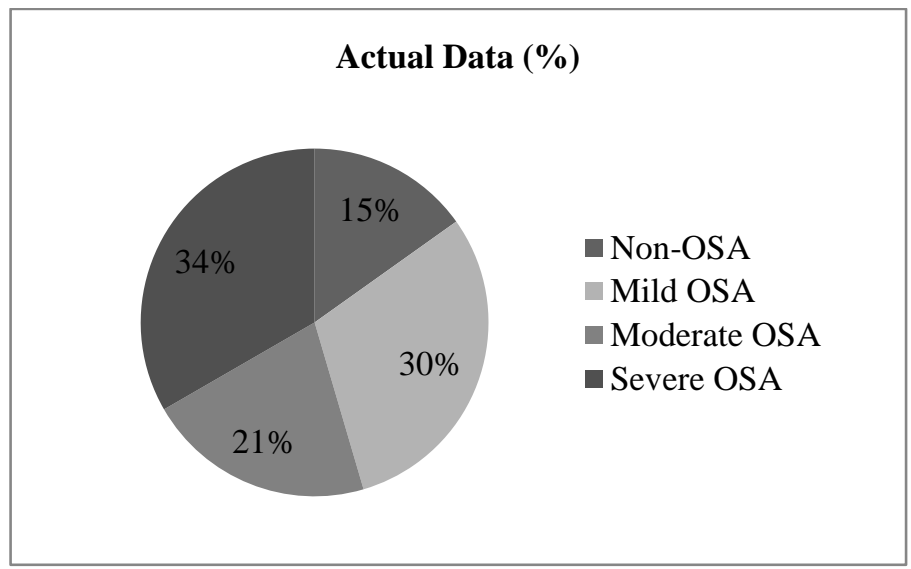

Fig. 2: Pie chat for actual subjects.

\section{Conclusion}

In this study, we have shown that multiclass classification of snoring subjects with different severity of OSA. The performance of the SVM classifier can be achieved on the sound spectrum features using K-means clustering, without needing to know the number of apneas. The performance evaluation of the classifier shows that non-OSA, mild OSA, moderate OSA, and severe OSA are classified at 60\%, 70\%, 57.14\%, and 100\% classification accuracy respectively. The overall accuracy is $75.76 \%$. The proposed method is an expedient tool for screening OSA as it provides a way of prioritizing patients for a second stage of diagnosis depending on their severity. The present study has some limitations. The results obtained need to use a database with a larger number of subjects. 


\section{Acknowledgements}

This work was supported by Prince of Songkla University Graduate Studies Grant as well as by Department of Electrical Engineering, Prince of Songkla University. The authors would like to thank sleep technician of Songklanagarind Hospital, Thailand for providing access to the respiratory sounds signals acquired in Sleep Disorders Service and Research Center.

\section{References}

[1] R. L. Wilkin, “Clinical Assessment in Respiratory Care,” St Louis, MO: Mosby, pp. 268-272, 1985.

[2] F. R. de Almeida, N. T. Ayas, R. Otsuka, H. Ueda, P. Hamilton, F. C. Ryan, and A. A. Lowe, "Nasal Pressure Recordings to Detect Obstructive Sleep Apnea," Sleep Breath, vol. 10, pp. 62-69, 2003.

[3] G. C. Chang and Y. P. Cheng, "Investigation of Noise Effect on Lung Sound Recognition," in Proceedings of the International Conference on Machine Learning and Cybernetics," Kunming, China, vol. 3, pp. 1298-1301, 2008.

[4] T. Young, L. Finn, et al., "Estimation of the Clinically Diagnosed Proportion of Sleep Apnea Syndrome in Middle Aged Men and Women," Sleep, vol. 20, pp. 705-706, 1997.

[5] J. V. Marcos, R. Hornero, D. Álvarez, F. del Campo, and C. Zamarón, "Assessment of Four Statistical Pattern Recognition Techniques to Assist in Obstructive Sleep Apnoea Diagnosis from Nocturnal Oximetry," Med. Eng. Phys., vol. 31, pp. 971-978, 2009.

[6] D. Pevernagie, R. Aarts, and M. D. Meyer, "The Acoustics of Snoring," Sleep Medicine Reviews, vol. 14, no. 2, pp. 131-144, 2009.

[7] D. Brunt, K. L. Lichstein, S. L. Noe, R. N. Aguillard, and K. W. Lester, "Intensity Pattern of Snoring Sounds as a Predictor for Sleep-Disordered Breathing," Sleep, vol. 20, pp. 1151-1156, 1997.

[8] K. Wilson, R. A. Stoohs, T. F. Mulrooney, L. J. Johnson, C. Guilleminault, and Z. Huang, "The Snoring Spectrum: Acoustic Assessment of Snoring Sound Intensity in 1,139 Individuals Undergoing Polysomnography," Chest, vol. 115, no. 3, pp. 762-70, 1999.

[9] J. A. Fiz, J. Abad, and J. Morera, et al., "Acoustic Analysis of Snoring Sound in Patients with Simple Snoring and Obstructive Sleep Apnoea,” Eur. Respir. J., vol. 9, pp. 2365-2370, 1996.

[10] J. R. Perez-Padilla, E. Slawinski, L. M. Difrancesco, R. R. Feige, J. E. Remmers, and W. A. Whitelaw, "Characteristics of the Snoring Noise in Patients with and without Occlusive Sleep Apnea," Am. Rev. Respir. Dis., vol. 147, pp. 635644, 1993.

[11] U. R. Abeyratne, A. S. Wakwella, and C. Hukins, "Pitch Jump Probability Measures for the Analysis of Snoring Sounds in Apnea," Physiol. Meas., vol. 26, no. 5, pp. 779-798, 2005.

[12] J. Sola-Soler, R. Jane, J. A. Fiz, and J. Morera, "Pitch Analysis in Snoring Signals from Simple Snorers and Patients with Obstructive Sleep Apnea," in Proceedings of the Annual Int. Conf. IEEE Eng. Med. Biol. Soc., vol. 2, pp. 15271528.

[13] A. K. Ng, T. S. Koh, E. Baey, and K. Puvanendran, "Diagnosis of Obstructive Sleep Apnea Using Formant Features of Snore Signals," in Proceedings of the IFBME World Congress Med. Phy. Biomed. Eng., vol. 14, pp. 861-864, 2006.

[14] W. W. Flemons, M. R. Littner, J. A. Rowley, P. Gay, W. M. Anderson, D. W. Hudgel, R. D. McEvoy, and D. I. Loube, "Home Diagnosis of Sleep Apnea: A Aystematic Review of the Literature; An Evidence Review Cosponsored by the American Academy of Sleep Medicine, the American College of Chest Physicians, and the American Thoracic Society," Chest, vol. 124, pp. 1543-1579, 2003.

[15] A. K. Jain, R. Duin, and J. Mao, "Statistical Pattern Recognition: A Review," IEEE Transactions on Pattern Analysis and Machine Intelligence, vol. 22, pp. 4-37, 2000.

[16] S. Lloyd, "Least Squares Quantization in PCM," IEEE Transactions on Information Theory, vol. 28, no. 2, pp. 129-137, 1982.

[17] C. Cortes and V. Vapnik, "Support-Vector Networks," Machine Learning, vol. 20, pp. 273-297, 1995.

[18] M. P. Paularj, S. Yaacob, M. S. bin Zanar Azalan, and R. Palaniappan, "A Phoneme Based Sign Language recognition system using skin color segmentation." in $6^{\text {th }}$ International Colloquium on Signal Processing and Its Applications (CSPA), pp. 1-5, 2010. 